\title{
A note on the solution of very basic vibrations problems obtained from a generalised situation
}

\author{
P. A. A. Laura (corresponding author), C. A. Rossit, D. V. Bambill and S. A. Vera \\ Institute of Applied Mechanics (IMA), Departments of Engineering and Physics, Universidad \\ Nacional del Sur, 8000 - Bahía Blanca, Argentina \\ E-mail: ima@criba.edu.ar
}

\begin{abstract}
Two forced vibrations problems are fundamental from academic and technological viewpoints; the suddenly applied constant-value force, and the situation where a sinusoidally varying excitation acts on the system. It is shown that both problems are particular cases of a more general excitation. The resonance stage is studied in detail.
\end{abstract}

Key words forced vibrations; constant-value force; sinusoidally varying excitation

\section{Introduction}

Consider a system with one degree of freedom subjected to a forced excitation:

$$
F(t)=F_{0} \quad t>0
$$

or

$$
F(t)=F_{0} \cos \omega t \quad t>0 \quad \omega \neq \omega_{n}=\sqrt{k / M}
$$

It is of interest that both cases are particular instances of the more general case:

$$
F(t)=F_{0} \mathrm{e}^{\alpha t} \cos \omega t
$$

where $\alpha$ is a real number. For the former case one has $\alpha=\omega=0$ and for the latter case $\alpha=0$.

When $\alpha$ and $\omega$ are not zero, no resonance occurs, as shown below. The approach is quite general and it may readily be extended to the case of viscous damping. It must be emphasized that the more general type of excitation described above corresponds, approximately, to the starting or stopping conditions for certain lathes and other machine-shop equipment, as has been determined experimentally by the authors.

\section{General solution}

Consider the problem

$$
M \ddot{x}+k x=F_{0} \mathrm{e}^{\alpha t} \cos \omega t
$$

subject to the intial conditions

$$
x(0)=\dot{x}(0)=0
$$


The consideration of more general initial conditions does not introduce any formal difficulties.

From equation 1 one obtains:

$$
\ddot{x}+\omega_{n}^{2} x=\frac{F_{0}}{M} \mathrm{e}^{\alpha t} \cos \omega t=F_{0}^{\prime} \mathrm{e}^{\alpha t} \cos \omega t
$$

The particular solution of equation 3 is of the type:

$$
x=A \mathrm{e}^{\alpha t} \cos \omega t+B \mathrm{e}^{\alpha t} \sin \omega t
$$

and substituting in equation 3 results in:

$$
\begin{gathered}
\mathrm{e}^{\alpha t} \cos \omega t\left[A\left(\alpha^{2}-\omega^{2}\right)+2 B \alpha \omega\right]+\mathrm{e}^{\alpha t} \sin \omega t\left[B\left(\alpha^{2}-\omega^{2}\right)-2 A \alpha \omega\right] \\
+\omega_{n}^{2} A \mathrm{e}^{\alpha t} \cos \omega t+\omega_{n}^{2} B \mathrm{e}^{\alpha t} \sin \omega t=F_{0}^{\prime} \mathrm{e}^{\alpha t} \cos \omega t
\end{gathered}
$$

Equating coefficients of similar terms one obtains:

$$
\begin{aligned}
& A\left(\alpha^{2}-\omega^{2}+\omega_{n}^{2}\right)+2 B \alpha \omega=F_{0}^{\prime} \\
& -2 A \alpha \omega+B\left(\alpha^{2}-\omega^{2}+\omega_{n}^{2}\right)=0
\end{aligned}
$$

which leads to:

$$
\begin{aligned}
& A=\frac{F_{0}}{M} \frac{\alpha^{2}-\omega^{2}+\omega_{n}^{2}}{\left(\alpha^{2}-\omega^{2}+\omega_{n}^{2}\right)^{2}+4 \alpha^{2} \omega^{2}} \\
& B=\frac{F_{0}}{M} \frac{2 \alpha \omega}{\left(\alpha^{2}-\omega^{2}+\omega_{n}^{2}\right)^{2}+4 \alpha^{2} \omega^{2}}
\end{aligned}
$$

Accordingly, the general solution to equation 3 is:

$$
x(t)=C_{1} \cos \omega_{n} t+C_{2} \sin \omega_{n} t+A \mathrm{e}^{\alpha t} \cos \omega t+B \mathrm{e}^{\alpha t} \sin \omega t
$$

Since

$$
x(0)=0=C_{t}+A
$$

Applying the second initial condition results in:

$$
\dot{x}(0)=0=C_{2} \omega_{n}+A \alpha+B \omega
$$

Then

$$
C_{2}=\frac{A \alpha+B \omega}{\omega_{n}}
$$

Finally one has:

$$
x(t)=-A \cos \omega_{n} t-\frac{A \alpha+B \omega}{\omega_{n}} \sin \omega_{n} t+A \mathrm{e}^{\alpha t} \cos \omega t+B \mathrm{e}^{\alpha t} \sin \omega t
$$




\section{Particular situations}

Case I: $\alpha=\omega=0$

Replacing in equation 7 one has:

$$
\begin{aligned}
& A=\frac{F_{0}}{M} \frac{\omega_{n}^{2}}{\omega_{n}^{4}}=\frac{F_{0}}{M} \frac{1}{\omega_{n}^{2}} \\
& B=0
\end{aligned}
$$

From equations 11 and 12:

$$
x(t)=-\frac{F_{0}}{M} \frac{1}{\omega_{n}^{2}} \cos \omega_{n} t+\frac{F_{0}}{M} \frac{1}{\omega_{n}^{2}}=\frac{F_{0}}{M} \frac{1}{\omega_{n}^{2}}\left(1-\cos \omega_{n} t\right)=\frac{F_{0}}{k}\left(1-\cos \omega_{n} t\right)
$$

Case II: $\alpha=0 ; \omega \neq \omega_{n}$

One has now:

$$
A=\frac{F_{0}}{M} \frac{-\omega^{2}+\omega_{n}^{2}}{\left(-\omega^{2}+\omega_{n}^{2}\right)^{2}}=\frac{F_{0}}{M} \frac{1}{\omega_{n}^{2}-\omega^{2}}=\frac{F_{0}}{M \omega_{n}^{2}} \frac{1}{1-\left(\frac{\omega}{\omega_{n}}\right)^{2}}=\frac{F_{0}}{k} \frac{1}{1-\left(\frac{\omega}{\omega_{n}}\right)^{2}}
$$

$$
B=0
$$

From equations 11 and 14 one obtains:

$$
x(t)=-\frac{F_{0}}{k} \frac{1}{1-\left(\frac{\omega}{\omega_{n}}\right)^{2}} \cos \omega_{n} t+\frac{F_{0}}{k} \frac{1}{1-\left(\frac{\omega}{\omega_{n}}\right)^{2}} \cos \omega t=\frac{F_{0}}{k} \frac{\cos \omega t-\cos \omega_{n} t}{1-\left(\frac{\omega}{\omega_{n}}\right)^{2}}
$$

Case III: $\alpha=0 ; \omega \rightarrow \omega_{n}$ Equation 15 is now indeterminate and the L'Hospital theorem yields:

$$
\begin{aligned}
x(t) & =-\left.\frac{F_{0}}{k} \frac{-t \sin \omega t}{-2\left(\frac{\omega}{\omega_{n}}\right) \frac{1}{\omega_{n}}}\right|_{\omega \rightarrow \omega_{n}}=\frac{F_{0}}{k} \frac{t \sin \omega_{n} t}{\frac{2}{\omega_{n}}}=\frac{1}{2} \frac{F_{0}}{k} \omega_{n} t \sin \omega_{n} t \\
& =\frac{1}{2} \frac{F_{0}}{k} \sqrt{\frac{k}{M}} t \sin \omega_{n} t=\frac{1}{2} \frac{F_{0}}{\sqrt{k M}} t \sin \omega_{n} t
\end{aligned}
$$

\section{General situation}

One immediately deduces from equations 7 and 11 that when $\alpha \neq 0$ the resonance phenomenon, as it is classically understood, does not appear.

If $\alpha=\omega$ one obtains:

$$
A=\frac{F_{0}}{M} \frac{\omega_{n}^{2}}{\omega_{n}^{4}+4 \omega^{4}}
$$




$$
B=\frac{F_{0}}{M} \frac{2 \omega^{2}}{\omega_{n}^{4}+4 \omega^{4}}
$$

If, in equation 17 , one makes $\nu=\nu_{n}$, one obtains:

$$
\begin{aligned}
& A=\frac{F_{0}}{M} \frac{\omega_{n}^{2}}{5 \omega_{n}^{4}}=\frac{1}{5} \frac{F_{0}}{k} \\
& B=\frac{2}{5} \frac{F_{0}}{M} \frac{1}{\omega_{n}^{2}}=\frac{2}{5} \frac{F_{0}}{k}
\end{aligned}
$$

\section{Concluding remarks}

As shown in equation 16 (case III), when the applied load possesses constant amplitude but varies sinusoidally, the response is amplified (over time) as the external frequency approaches the natural frequency of the mechanical system. This is the classical case of resonance.

One the other hand, when one deals with a step loading (case I) the response is amplified by the factor $\left(1-\cos \omega_{n} t\right)$ and the maximum value of the amplification factor is $2\left(t=\pi / \omega_{n}\right)$.

Finally, for the general situation where $\alpha \neq 0$, the response is given by equation 11, where $A$ and $B$ are defined in equation 7. As the time increases the amplitude also increases for $\alpha>0$ in an indefinite fashion. When $\alpha$ is negative, equation 11 yields as a limiting, functional value:

$$
x(t)=-A \cos \omega_{n} t-\frac{A \alpha+B \omega}{\omega_{n}} \sin \omega_{n} t
$$

When $\omega=\omega_{n}$ it can be shown that $A$ and $B$ acquire definite, finite values (see equation 7).

\section{Acknowledgements}

The study was sponsored by CONICET, Secretaria General de Ciencia y Tecnología of Universidad Nacional del Sur and Rocca Foundation (Techint). The authors are indebted to Dr C. M. Leech, Editor of IJMEE, and to an anonymous referee for their constructive criticism and kind remarks, which allowed the authors to improve considerably the present paper. 